PROCEEDINGS OF THE

AMERICAN MATHEMATICAL SOCIETY

Volume 133, Number 7, Pages 2013-2021

S 0002-9939(05)07748-8

Article electronically published on January 14, 2005

\title{
MEROMORPHIC FUNCTIONS AND FACTORIALITY
}

\author{
W. KUCHARZ
}

(Communicated by Michael Stillman)

\begin{abstract}
Let $K$ be a compact subset of a connected Stein manifold $X$. We study algebraic properties of the ring of meromorphic functions on $X$ without poles in $K$.
\end{abstract}

\section{Introduction AND MAIN RESUlts}

Let $X$ be a connected Stein manifold. Denote by $\mathcal{O}(X)$ (resp. $\mathcal{O}_{x}$ ) the ring of holomorphic functions on $X$ (resp. the ring of germs of holomorphic functions at a point $x$ in $X)$. We regard $\mathcal{O}(X)$ as a subring of the field $\mathcal{M}(X)$ of meromorphic functions on $X$. Since $X$ is a Stein manifold, $\mathcal{M}(X)$ is the field of fractions of $\mathcal{O}(X)$; cf. [9, Theorem 7.4.6]. Given a compact subset $K$ of $X$, we let

$$
\mathcal{M}_{K}(X)
$$

denote the subring of $\mathcal{M}(X)$ consisting of all meromorphic functions on $X$ with no poles in $K$. In this paper we investigate algebraic properties of $\mathcal{M}_{K}(X)$. Some mild assumptions on $K$ allow us to prove that the ring $\mathcal{M}_{K}(X)$ is regular (Theorem 1.1) and to give necessary and sufficient conditions for $\mathcal{M}_{K}(X)$ to be a unique factorization domain (Theorem 1.2). If $\operatorname{dim} X=1$, then $\mathcal{M}_{K}(X)$ is always a regular ring and a unique factorization domain (Corollary 1.3). Assuming that $X$ is an algebraic subset of $\mathbb{C}^{N}$, for some $N$, the $\operatorname{ring} \mathcal{M}_{K}(X)$ is a unique factorization domain for every compact subset $K$ of $X$ if and only if $H^{2}(X, \mathbb{Z})=0$ (Theorem 1.4). The reader may consult [2, 5, 7, 13] for related results concerning the ring of germs at $K$ of holomorphic functions defined in a neighborhood of $K$.

We need some preparation in order to state precisely our results. Denote by $\hat{K}$ the holomorphic hull of $K$ in $X$,

$$
\hat{K}=\left\{x \in X|| f(x)\left|\leq \sup _{y \in K}\right| f(y) \mid \text { for every } f \text { in } \mathcal{O}(X)\right\} .
$$

Since $X$ is a Stein manifold, $\hat{K}$ is compact. Recall that $K$ is said to be holomorphically convex in $X$ if $K=\hat{K}$. Of course, $K \subseteq \hat{K}$ and $\hat{K}$ is holomorphically convex in $X$.

We write $H^{*}(-, \mathbb{Z})$ to denote the Čech cohomology with coefficients in $\mathbb{Z}$. Let

$$
G(K)
$$

Received by the editors April 17, 2003 and, in revised form, February 26, 2004.

2000 Mathematics Subject Classification. Primary 32A20, 32A38.

This paper was written at the Max-Planck-Institut für Mathematik in Bonn, whose support and hospitality are gratefully acknowledged. 
denote the image of the restriction homomorphism $H^{2}(X, \mathbb{Z}) \rightarrow H^{2}(K, \mathbb{Z})$ (that is, the homomorphism induced by the inclusion map $K \hookrightarrow X)$. The restriction homomorphism $H^{2}(\hat{K}, \mathbb{Z}) \rightarrow H^{2}(K, \mathbb{Z})$ gives rise to a homomorphism from $G(\hat{K})$ onto $G(K)$, written

$$
\alpha_{K}: G(\hat{K}) \rightarrow G(K),
$$

whose kernel will be denoted by $\hat{G}(K)$,

$$
\hat{G}(K)=\operatorname{Ker} \alpha_{\mathrm{K}} \text {. }
$$

The groups $G(K)$ and $\hat{G}(K)$ will play an important role. Note that $\hat{G}(K)=0$ if $K$ is holomorphically convex in $X$. Furthermore, $G(K)=0$ and $\hat{G}(K)=0$, provided $H^{2}(X, \mathbb{Z})=0$.

Let us set

$$
S(K)=\left\{f \in \mathcal{O}(X) \mid f^{-1}(0) \subseteq X \backslash K\right\} .
$$

Clearly, $S(K)$ is a multiplicatively closed subset of the $\operatorname{ring} \mathcal{O}(X)$. The $\operatorname{ring} \mathcal{O}_{K}(X)$ of fractions of $\mathcal{O}(X)$ with denominators in $S(K)$ is a subring of $\mathcal{M}(X)$. We have

$$
\mathcal{O}_{K}(X) \subseteq \mathcal{M}_{K}(X) .
$$

Theorem 1.1. For any compact subset $K$ of $X$, the ring $\mathcal{O}_{K}(X)$ is regular. If $\hat{G}(K)=0$, then $\mathcal{O}_{K}(X)=\mathcal{M}_{K}(X)$.

Let us recall that a commutative ring with identity is said to be regular if it is Noetherian and its localization at each maximal ideal is a regular local ring (cf. [10, p. 140]). It is well known that a regular ring $A$ with no zero divisors is a unique factorization domain if and only if $\operatorname{Pic}(A)=0$, where $\operatorname{Pic}(A)$ is the Picard group of $A$ (the group of isomorphism classes of finitely generated projective $A$-modules of rank 1); cf. [4] or, more precisely, see the references to [4] given in [3, pp. 306, 307] and [10, p. 142, Theorem 48].

Theorem 1.2. Assume that $\hat{G}(K)=0$. Then $\operatorname{Pic}\left(\mathcal{M}_{K}(X)\right)$ is canonically isomorphic to $G(K)$. In particular, $\mathcal{M}_{K}(X)$ is a unique factorization domain if and only if $G(K)=0$.

Specializing to $\operatorname{dim} X=1$, we obtain the following.

Corollary 1.3. If $\operatorname{dim} X=1$, then for any compact subset $K$ of $X$, the ring $\mathcal{M}_{K}(X)$ is regular and a unique factorization domain.

Proof. Since $\operatorname{dim} X=1$, we have $H^{2}(X, \mathbb{Z})=0$. Hence $G(K)=0$ and $\hat{G}(K)=0$. The conclusion follows from Theorems 1.1 and 1.2.

Let us also mention that if $\operatorname{dim} X \geq 1$, then the $\operatorname{ring} \mathcal{O}(X)$ is not Noetherian and not a unique factorization domain.

Theorem 1.4. Let $X$ be a nonsingular irreducible algebraic subset of $\mathbb{C}^{N}$ for some $N$. Then the following conditions are equivalent:

(a) for every compact subset $K$ of $X$, the ring $\mathcal{M}_{K}(X)$ is a unique factorization domain,

(b) for every compact holomorphically convex subset $K$ of $X$, the ring $\mathcal{M}_{K}(X)$ is a unique factorization domain,

(c) $H^{2}(X, \mathbb{Z})=0$. 


\section{Proofs}

We shall frequently use the fact that any compact holomorphically convex subset of a Stein manifold has a base of neighborhoods consisting of open Stein submanifolds; cf. [9, Theorems 5.1.6, 5.2.10].

For any point $x$ in $X$,

$$
m_{x}=\{f \in \mathcal{O}(X) \mid f(x)=0\}
$$

is a maximal ideal of the ring $\mathcal{O}(X)$ (not every maximal ideal of $\mathcal{O}(X)$ is of this form). Given a compact subset $K$ of $X$, we shall now regard $\mathcal{O}(X)$ as a subring of $\mathcal{O}_{K}(X)$. Clearly, if $x$ is a point in $K$, then $m_{x} \mathcal{O}_{K}(X)$ is a maximal ideal of $\mathcal{O}_{K}(X)$.

Lemma 2.1. Let $m$ be a maximal ideal of the ring $\mathcal{O}_{K}(X)$. If $K$ is holomorphically convex in $X$, then

for some point $x$ in $K$.

$$
m=m_{x} \mathcal{O}_{K}(X)
$$

Proof. It suffices to show that there is a point $x$ in $K$ such that $f(x)=0$ for all $f$ in $m \cap \mathcal{O}(X)$. Suppose that this assertion does not hold. Then one can find functions $f_{1}, \ldots, f_{r}$ in $m \cap \mathcal{O}(X)$ which have no common zero in a neighborhood $U$ of $K$ in $X$. We may assume that $U$ is an open Stein neighborhood.

Let $\mathcal{J}$ be the sheaf of ideals on $X$ generated by $f_{1}, \ldots, f_{r}$,

$$
\mathcal{J}_{x}=\left(f_{1}, \ldots, f_{r}\right) \mathcal{O}_{x}
$$

for all $x$ in $X$. Clearly, 1 belongs to $\Gamma(U, \mathcal{J})$. Hence there is an $f$ in $\Gamma(X, \mathcal{J})$ such that $f$ is close to 1 on $K$; cf. [9, Theorem 7.2.7]. In particular, $f$ is in $S(K)$ and, by [9] Theorem 7.2.9], $f$ is also in the ideal $\left(f_{1}, \ldots, f_{r}\right) \mathcal{O}(X)$. It follows that $f$ is in $S(K) \cap m$. This implies $m=\mathcal{O}_{K}(X)$, a contradiction.

Denote by $\mathcal{O}_{K}$ the ring of germs at $K$ of holomorphic functions defined in a neighborhood of $K$. Note the canonical ring homomorphisms $\mathcal{O}(X) \rightarrow \mathcal{O}_{K}$ and $\mathcal{O}_{K}(X) \rightarrow \mathcal{O}_{K}$.

Lemma 2.2. If $K$ is holomorphically convex in $X$, then the ring $\mathcal{O}_{K}$ is faithfully flat over $\mathcal{O}_{K}(X)$.

Proof. We shall first show that $\mathcal{O}_{K}$ is flat over $\mathcal{O}(X)$. To this end we shall use a characterization of flatness in terms of solutions of linear equations [10, p. 17, Theorem 1]. Given $f_{1}, \ldots, f_{r}$ in $\mathcal{O}(X)$ and $g_{1}, \ldots, g_{r}$ in $\mathcal{O}_{K}$ with

$$
f_{1} g_{1}+\cdots+f_{r} g_{r}=0 \text { in } \mathcal{O}_{K}
$$

we have to show the existence of $f_{i j}$ in $\mathcal{O}(X)$ and $h_{j}$ in $\mathcal{O}_{K}, 1 \leq i \leq r, 1 \leq j \leq s$ for some positive integer $s$, such that

$$
f_{1} f_{1 j}+\cdots+f_{r} f_{r j}=0 \text { in } \mathcal{O}(X) \text { for all } 1 \leq j \leq s
$$

and

$$
g_{i}=f_{i 1} h_{1}+\cdots+f_{i s} h_{s} \text { in } \mathcal{O}_{K} \text { for all } 1 \leq i \leq r .
$$

We proceed as follows. Let $\mathcal{R}$ be the sheaf of relations among $f_{1}, \ldots, f_{r}$,

$$
\mathcal{R}_{x}=\left\{\left(a_{1}, \ldots, a_{r}\right) \in \mathcal{O}_{x}^{r} \mid f_{1} a_{1}+\cdots+f_{r} a_{r}=0 \text { in } \mathcal{O}_{x}\right\}
$$

for all $x$ in $X$. By Oka's theorem [9, Theorem 6.4.1], $\mathcal{R}$ is a coherent sheaf, and hence it follows from Cartan's Theorem A [9, Theorem 7.2.8] that there exist global 
sections $F_{1}, \ldots, F_{s}$ of $\mathcal{R}$ which generate each stalk $\mathcal{R}_{x}$ for $x$ in an open neighborhood $U$ of $K$ in $X$. Since $K$ is holomorphically convex in $X$, we may assume that $U$ is Stein and the germs $g_{1}, \ldots, g_{r}$ have holomorphic representatives on $U$. Hence writing $G=\left(g_{1}, \ldots, g_{r}\right)$, we obtain

$$
G=h_{1} F_{1}+\cdots+h_{s} F_{s}
$$

for some $h_{1}, \ldots, h_{s}$ in $\mathcal{O}_{K}$; cf. [9, Theorem 7.2.9]. Setting $F_{j}=\left(f_{1 j}, \ldots, f_{r j}\right)$ for $1 \leq j \leq s$, we see that (1) and (2) are satisfied. It follows that $\mathcal{O}_{K}$ is flat over $\mathcal{O}(X)$.

It immediately follows that $\mathcal{O}_{K}$ is flat over $\mathcal{O}_{K}(X)$. In order to prove that $\mathcal{O}_{K}$ is faithfully flat over $\mathcal{O}_{K}(X)$ it suffices to demonstrate that given a maximal ideal $m$ of $\mathcal{O}_{K}(X)$, we have $m \mathcal{O}_{K} \neq \mathcal{O}_{K}$; cf. [10, p. 25, Theorem 2]. By Lemma $2.1, m=m_{x} \mathcal{O}_{K}(X)$ for some point $x$ in $K$. Clearly, $m \mathcal{O}_{K}=m_{x} \mathcal{O}_{K}$. Since $m_{x} \mathcal{O}_{K} \neq \mathcal{O}_{K}$, the proof is complete.

Proposition 2.3. For any compact subset $K$ of $X$, the ring $\mathcal{O}_{K}(X)$ is regular.

Proof. Step 1. Assume that $K$ is a semianalytic compact holomorphically convex subset of $X$. It follows that the ring $\mathcal{O}_{K}$ is Noetherian [7, Théorème (I, 9)]. By Lemma 2.2, $\mathcal{O}_{K}(X)$ is a Noetherian ring; cf. [4, Chapitre I, p. 50].

In order to complete Step 1 it remains to show that for each maximal ideal $m$ of $\mathcal{O}_{K}(X)$, the localization $\mathcal{O}_{K}(X)_{m}$ of $\mathcal{O}_{K}(X)$ at $m$ is a regular local ring. By Lemma 2.1, $m=m_{x} \mathcal{O}_{K}(X)$ for some point $x$ in $K$. We have $S(K) \cap m_{x}=\emptyset$, which implies that the local rings $\mathcal{O}_{K}(X)_{m}$ and $\mathcal{O}(X)_{m_{x}}$ are isomorphic. Hence it suffices to prove that the local ring $\mathcal{O}(X)_{m_{x}}$ is regular. We have $\mathcal{O}(X)_{m_{x}}=\mathcal{O}_{\{x\}}(X)$, and hence the ring $\mathcal{O}(X)_{m_{x}}$ is Noetherian (the set $\{x\}$ being semianalytic and holomorphically convex in $X)$. Since $X$ is a Stein manifold, there exist $f_{1}, \ldots, f_{n}$ in $\mathcal{O}(X)$, where $n=\operatorname{dim} X$, such that $\left(f_{1}, \ldots, f_{n}\right) \mathcal{O}_{x}$ is the maximal ideal of $\mathcal{O}_{x}$; cf. [9] Definition 5.1.3 $(\gamma)$ ]. By Lemma 2.2, $\mathcal{O}_{x}$ is flat over $\mathcal{O}(X)_{m_{x}}$ and therefore $\left(f_{1}, \ldots, f_{n}\right) \mathcal{O}(X)_{m_{x}}$ is the maximal ideal of $\mathcal{O}(X)_{m_{x}}$. It readily follows that the completions of $\mathcal{O}(X)_{m_{x}}$ and $\mathcal{O}_{x}$ with respect to their maximal ideals are isomorphic. Since $\mathcal{O}_{x}$ is a regular local ring, so is $\mathcal{O}(X)_{m_{x}}$; cf. [10 p. 175].

Step 2. Let $K$ be an arbitrary compact subset of $X$. We may assume that $X$ is a closed submanifold of $\mathbb{C}^{N}$ for some $N$. Choose a closed ball $B$ in $\mathbb{C}^{N}$ containing $K$. Clearly, the compact subset $C=B \cap X$ of $X$ is semianalytic and holomorphically convex in $X$. According to Step 1, the ring $\mathcal{O}_{C}(X)$ is regular. Since $S(C) \subseteq S(K)$, the ring $\mathcal{O}_{K}(X)$ is a localization of $\mathcal{O}_{C}(X)$, and hence $\mathcal{O}_{K}(X)$ is also a regular ring.

In what follows we shall make use several times of the following consequence of Cartan's Theorem B: For any Stein manifold $Y$,

$$
c_{1}: \operatorname{Pic}(Y) \rightarrow H^{2}(Y, \mathbb{Z})
$$

is an isomorphism, where $\operatorname{Pic}(Y)$ is the group of isomorphism classes of holomorphic line bundles on $Y$ and $c_{1}$ is the first Chern class homomorphism; cf. [8, p. 197, Theorem C]. In particular, any holomorphic line bundle on $Y$ is holomorphically trivial if and only if it is topologically trivial.

Proof of Theorem 1.1. In view of Proposition 2.3 and the inclusion $\mathcal{O}_{K}(X) \subseteq$ $\mathcal{M}_{K}(X)$ it suffices to show $\mathcal{M}_{K}(X) \subseteq \mathcal{O}_{K}(X)$. Let $\varphi$ be in $\mathcal{M}_{K}(X) \backslash\{0\}$. To 
show that $\varphi$ is in $\mathcal{O}_{K}(X)$ we proceed as follows. For each point $x$ in $X$,

$$
\mathcal{J}_{x}=\left\{h \in \mathcal{O}_{x} \mid h \varphi \text { is in } \mathcal{O}_{x}\right\}
$$

is a nonzero ideal of the $\operatorname{ring} \mathcal{O}_{x}$. It is well known that the union $\mathcal{J}$ of all $\mathcal{J}_{x}$ is a coherent sheaf of principal ideals on $X$; cf. 9, proof of Theorem 7.4.6]. There is a family $\left\{U_{i}, h_{i}\right\}_{i \in I}$, where $\left\{U_{i}\right\}_{i \in I}$ is an open cover of $X$ and $h_{i}: U_{i} \rightarrow \mathbb{C}$ is a holomorphic function generating $\mathcal{J} \mid U_{i}$ for each $i$ in $I$. On $U_{i} \cap U_{j}$ we have $h_{i}=g_{i j} h_{j}$, where $g_{i j}: U_{i} \cap U_{j} \rightarrow \mathbb{C} \backslash\{0\}$ is a holomorphic function. Note that $\left\{U_{i}, h_{i}\right\}_{i \in I}$ describes the divisor of poles of $\varphi$. Let $L$ be the holomorphic line bundle on $X$ determined by $\left\{U_{i}, g_{i j}\right\}$ and let $s: X \rightarrow L$ be the holomorphic section corresponding to $\left\{h_{i}\right\}_{i \in I}$. Note

$$
s^{-1}(0) \subseteq X \backslash K
$$

Consider the dual line bundle $L^{\vee}$ on $X$. In view of (1), the restriction $L^{\vee} \mid K$ is topologically trivial on $K$ and hence $c_{1}\left(L^{\vee} \mid K\right)=0$ in $H^{2}(K, \mathbb{Z})$, where $c_{1}(-)$ stands for the first Chern class. Since $c_{1}\left(L^{\vee} \mid \hat{K}\right)$ is in $G(\hat{K}), \alpha_{K}\left(c_{1}\left(L^{\vee} \mid \hat{K}\right)\right)=c_{1}\left(L^{\vee} \mid K\right)$, and $\operatorname{Ker} \alpha_{K}=\hat{G}(K)=0$, we get $c_{1}\left(L^{\vee} \mid \hat{K}\right)=0$. The last equality implies that $L^{\vee} \mid \hat{K}$ is topologically trivial on $\hat{K}$. Choose an open neighborhood $U$ of $\hat{K}$ in $X$ such that $L^{\vee} \mid U$ is topologically trivial. We may assume that $U$ is Stein, and hence $L^{\vee} \mid U$ is holomorphically trivial. By [9, Theorem 7.2.7], there exists a holomorphic section $u: X \rightarrow L^{\vee}$ satisfying

$$
u^{-1}(0) \subseteq X \backslash \hat{K}
$$

Define $g: X \rightarrow \mathbb{C}$ by

$$
g(x)=u(x)(s(x)) \text { for all } x \text { in } X .
$$

Clearly, $g$ is a holomorphic function and moreover $g$ belongs to $\Gamma(X, \mathcal{J})(s$ is locally $h_{i}$ and $u$ acts on $s$ by multiplying $h_{i}$ by a holomorphic function). Hence $f=g \varphi$ is in $\mathcal{O}(X)$. Combining (1) and (2), we get $g^{-1}(0) \subseteq X \backslash K$, and hence $\varphi=f / g$ belongs to $\mathcal{O}_{K}(X)$.

For the proof of Theorem 1.2 we need another result.

Theorem 2.4. Let $Y$ be a Stein manifold. If $E$ is a holomorphic vector bundle on $Y$, then the $\mathcal{O}(Y)$-module $\Gamma(Y, E)$ of global holomorphic sections of $E$ is finitely generated and projective. Furthermore, the correspondence $E \rightarrow \Gamma(Y, E)$ is an equivalence between the category of holomorphic vector bundles on $Y$ and the category of finitely generated projective $\mathcal{O}(Y)$-modules.

Reference for the proof. [6, Satz 6.2 and Satz 6.3].

Henceforth, when no confusion is possible, we shall identify modules and their isomorphism classes.

Proof of Theorem 1.2. Let $A$ be a compact subset of $X$. Denote by

$$
\beta_{A}: \operatorname{Pic}\left(\mathcal{O}_{A}(X)\right) \rightarrow \operatorname{Pic}\left(\mathcal{O}_{A}\right)
$$

the group homomorphism induced by the canonical ring homomorphism $\mathcal{O}_{A}(X) \rightarrow$ $\mathcal{O}_{A}$. We assert

$$
\beta_{A} \text { is injective if } A \text { is holomorphically convex in } X \text {. }
$$


Indeed, endow $\mathcal{O}_{A}$ with the topology of uniform convergence on $A$. If $A$ is holomorphically convex in $X$, then the image of $\mathcal{O}(X)$ under the canonical homomorphism $\mathcal{O}(X) \rightarrow \mathcal{O}_{A}$ is dense in $\mathcal{O}_{A}$; cf. [9, Theorem 7.2.7]. Hence (1) is a consequence of [15, Theorem 2.2].

We shall now define a canonical homomorphism

$$
\gamma_{A}: \operatorname{Pic}\left(\mathcal{O}_{A}\right) \rightarrow H^{2}(A, \mathbb{Z}) .
$$

Denote by $\mathcal{C}(A)$ the ring of all continuous functions from $A$ into $\mathbb{C}$, and let

$$
\delta_{A}: \operatorname{Pic}\left(\mathcal{O}_{A}\right) \rightarrow \operatorname{Pic}(\mathcal{C}(A))
$$

be the group homomorphism induced by the canonical ring homomorphism $\mathcal{O}_{A} \rightarrow$ $\mathcal{C}(A)$. Denote by

$$
\eta_{A}: \operatorname{Pic}(\mathcal{C}(A)) \rightarrow H^{2}(A, \mathbb{Z})
$$

the homomorphism which assigns to each element $Q$ of $\operatorname{Pic}(\mathcal{C}(A))$ the first Chern class of the topological complex line bundle on $A$ determined by $Q$; cf. [14. Note that $\eta_{A}$ is an isomorphism. By definition, $\gamma_{A}$ is the composite of $\delta_{A}$ and $\eta_{A}$, that is $\gamma_{A}=\eta_{A} \circ \delta_{A}$. We claim

$$
\gamma_{A} \text { is injective if } A \text { is holomorphically convex in } X \text {. }
$$

In order to prove (2) note that

$$
\mathcal{O}_{A}=\text { ind } \lim \mathcal{O}(U),
$$

where $U$ runs through the family of open Stein neighborhoods of $A$ in $X$. It follows that

$$
\operatorname{Pic}\left(\mathcal{O}_{A}\right)=\operatorname{ind} \lim \operatorname{Pic}(\mathcal{O}(U))
$$

(see for example [15, Lemma 7.2]). By Theorem 2.4, every element of $\operatorname{Pic}(\mathcal{O}(U)$ ) is of the form $\Gamma(U, L)$ for some holomorphic line bundle $L$ on $U$. Suppose $c_{1}(L \mid A)=0$. Then $L \mid A$ is topologically trivial on $A$. There is an open neighborhood $U^{\prime}$ of $A$ in $U$ such that $L \mid U^{\prime}$ is topologically trivial on $U^{\prime}$. We may choose $U^{\prime}$ to be Stein, and hence $L \mid U^{\prime}$ is holomorphically trivial on $U^{\prime}$. It follows that $\Gamma(U, L)$ represents 0 in $\operatorname{Pic}\left(\mathcal{O}_{A}\right)$. Thus $(2)$ is proved.

Define

$$
c_{A}: \operatorname{Pic}\left(\mathcal{O}_{A}(X)\right) \rightarrow H^{2}(A, \mathbb{Z})
$$

by setting $c_{A}=\gamma_{A} \circ \beta_{A}$. In view of (1) and (2), we have

$$
c_{A} \text { is injective if } A \text { is holomorphically convex in } X \text {. }
$$

If $B$ is a compact subset of $X$ containing $A$, then the following diagram is commutative:

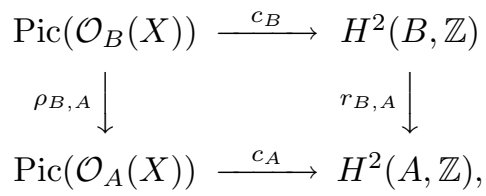

where $r_{B, A}$ is the restriction homomorphism and $\rho_{B, A}$ is the homomorphism induced by the inclusion $\mathcal{O}_{B}(X) \rightarrow \mathcal{O}_{A}(X)$. Since $S(B) \subseteq S(A)$, the ring $\mathcal{O}_{A}(X)$ is a localization of $\mathcal{O}_{B}(X)$. By Proposition 2.3, $\mathcal{O}_{B}(X)$ is a regular ring and hence

$$
\rho_{B, A} \text { is surjective; }
$$

cf. [1. p. 144, Proposition 7.17, p. 147, Theorem 7.21]. 
We shall now show that

$$
c_{A}\left(\operatorname{Pic}\left(\mathcal{O}_{A}(X)\right)\right) \subseteq G(A) .
$$

This can be done as follows. We may assume that $X$ is a closed submanifold of $\mathbb{C}^{N}$ for some $N$. Let $\left\{B_{i}\right\}_{i \geq 1}$ be a sequence of closed balls in $\mathbb{C}^{N}$ such that $A \subseteq B_{1} \cap X$, $B_{i} \subseteq B_{i+1}$, and $X$ is the union of the $B_{i} \cap X$ for $i \geq 1$. Let us set $A_{0}=A$ and $A_{i}=B_{i} \cap X$ for $i \geq 1$. Clearly, $A_{i}$ is semianalytic and holomorphically convex in $X$ for $i \geq 1$. There is a triangulation of $X$ for which each $A_{i}$ with $i \geq 1$ is a compact polyhedron [12, Theorem II.2.1'].

Let $P$ be in $\operatorname{Pic}\left(\mathcal{O}_{A}(X)\right)$. We define recursively a sequence $\left\{P_{i}\right\}_{i \geq 0}$, where $P_{i}$ belongs to $\operatorname{Pic}\left(\mathcal{O}_{A_{i}}(X)\right)$. Set $P_{0}=P$. Suppose that $P_{i}$ in $\operatorname{Pic}\left(\mathcal{O}_{A_{i}}(X)\right)$ is already defined, and let $P_{i+1}$ be an element of $\operatorname{Pic}\left(\mathcal{O}_{A_{i+1}}(X)\right)$ which is sent to $P_{i}$ by the homomorphism

$$
\rho_{A_{i+1}}, A_{i}: \operatorname{Pic}\left(\mathcal{O}_{A_{i+1}}(X)\right) \rightarrow \operatorname{Pic}\left(\mathcal{O}_{A_{i}}(X)\right)
$$

(by (5), this homomorphism is surjective).

The restriction homomorphisms

$$
r_{A_{i+1}}, A_{i}: H^{2}\left(A_{i+1}, \mathbb{Z}\right) \rightarrow H^{2}\left(A_{i}, \mathbb{Z}\right),
$$

for $i \geq 0$, give rise to the projective limit

$$
\operatorname{proj} \lim H^{2}\left(A_{i}, \mathbb{Z}\right) \text {. }
$$

In view of (4), $\left(c_{A_{0}}\left(P_{0}\right), c_{A_{1}}\left(P_{1}\right), c_{A_{2}}\left(P_{2}\right), \ldots\right)$ belongs to this limit. Since the canonical homomorphism

$$
H^{2}(X, \mathbb{Z}) \rightarrow \operatorname{proj} \lim H^{2}\left(A_{i}, \mathbb{Z}\right)
$$

is surjective [11, Lemma 2], it follows that $c_{A}(P)=c_{A_{0}}\left(P_{0}\right)$ is in $G(A)$. This completes the proof of (6).

Our next step is the proof of

$$
G(A) \subseteq c_{A}\left(\operatorname{Pic}\left(\mathcal{O}_{A}(X)\right)\right) .
$$

Let $u$ be in $G(A)$. Choose a cohomology class $v$ in $H^{2}(X, \mathbb{Z})$ which is sent to $u$ by the restriction homomorphism $H^{2}(X, \mathbb{Z}) \rightarrow H^{2}(A, \mathbb{Z})$. There is a holomorphic line bundle $M$ on $X$ with $c_{1}(M)=v$. By Theorem 2.4, $S(A)^{-1} \Gamma(X, M)$ is in $\operatorname{Pic}\left(\mathcal{O}_{A}(X)\right)$ and by definition of $c_{A}$, we have $c_{A}\left(S(A)^{-1} \Gamma(X, M)\right)=u$. Hence $(7)$ is proved.

Let

$$
\bar{c}_{A}: \operatorname{Pic}\left(\mathcal{O}_{A}(X)\right) \rightarrow G(A)
$$

be the surjective homomorphism determined by $c_{A}\left(\bar{c}_{A}\right.$ is just $c_{A}$ regarded as a homomorphism onto its image; cf. (6) and (7)).

After this preparation we are ready to complete the proof of the theorem. We shall now show that

$$
\bar{c}_{K}: \operatorname{Pic}\left(\mathcal{O}_{K}(X)\right) \rightarrow G(K) \text { is an isomorphism. }
$$

Since $\bar{c}_{K}$ is a surjective homomorphism, it remains to demonstrate injectivity of $\bar{c}_{K}$. Diagram (4), with $A=K$ and $B=\hat{K}$, and (8) yield the following commutative 
diagram:

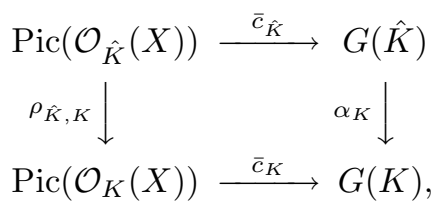

where $\alpha_{K}$ is the restriction of $r_{\hat{K}, K}$ (cf. Section 1). According to (3), $\bar{c}_{\hat{K}}$ is injective. Recall that $\hat{G}(K)=\operatorname{Ker} \alpha_{K}=0$, and hence $\alpha_{K}$ is injective. Since by (5), $\rho_{\hat{K}, K}$ is surjective, we obtain that $\bar{c}_{K}$ is injective. Thus (9) is proved, and the groups $\operatorname{Pic}\left(\mathcal{O}_{K}(X)\right)$ and $G(K)$ are canonically isomorphic.

By Theorem 1.1, the ring $\mathcal{O}_{K}(X)=\mathcal{M}_{K}(X)$ is regular, and therefore the proof is complete.

Proof of Theorem 1.4. If (c) holds, then $\hat{G}(K)=0$ and $G(K)=0$ for every compact subset $K$ of $X$, and hence (a) is satisfied in view of Theorem 1.2. It is obvious that (a) implies (b). It remains then to show that (b) implies (c).

Suppose (b) holds. Choose a compact subset $C$ of $X$ such that the inclusion map $C \hookrightarrow X$ is a homotopy equivalence; cf. for example [3, Corollary 9.3.7]. The restriction homomorphism $H^{2}(X, \mathbb{Z}) \rightarrow H^{2}(C, \mathbb{Z})$ is an isomorphism. It follows that for $K=\hat{C}$ the restriction homomorphism $H^{2}(X, \mathbb{Z}) \rightarrow H^{2}(K, \mathbb{Z})$ is injective (note $C \subseteq K)$. This implies that $H^{2}(X, \mathbb{Z})$ is isomorphic to $G(K)$. Since $K$ is holomorphically convex in $X$, we have $\hat{G}(K)=0$ and hence, by Theorem 1.2 and condition (b), $G(K)=0$. Therefore $H^{2}(X, \mathbb{Z})=0$ and (c) is satisfied.

\section{REFERENCES}

[1] H. Bass, Algebraic K-Theory, New York, Benjamin, 1968. MR0249491 (40:2736)

[2] J. Bochnak, Sur la factorialité des anneaux de fonctions analytiques, C. R. Acad. Sci. Paris Sér. A 279 (1974), 269-272. MR0377100 (51:13274)

[3] J. Bochnak, M. Coste and M.-F. Roy, Real Algebraic Geometry, Ergebnisse der Math. und ihrer Grenzgeb. Folge 3, Vol. 36, Berlin Heidelberg New York, Springer, 1998. MR 1659509 (2000a:14067)

[4] N. Bourbaki, Algèbre Commutative, Paris, Hermann, 1961-1965. MR0217051 (36:146). MR0171800 (30:2027) MR0194450 (33:2660) MR0260715 (41:5339)

[5] H. Dales, The ring of holomorphic functions on a Stein compact set as a unique factorization domain, Proc. Amer. Math. Soc. 44 (1974), 88-92. MR.0333245 (48:11570)

[6] O. Forster, Zur Theorie der Steinschen Algebren und Moduln, Math. Z. 97 (1967), 376-405. MR0213611 (35:4469)

[7] J. Frisch, Points de platitude d'un morphisme d'espaces analytiques complexes, Invent. Math. 4 (1967), 118-138. MR0222336 (36:5388)

[8] P. Griffiths and J. Adams, Topics in Algebraic and Analytic Geometry, Math. Notes, Vol. 13, Princeton Univ. Press, Princeton, New Jersey, 1974. MR0355119 (50:7596)

[9] L. Hörmander, An Introduction to Complex Analysis in Several Variables, Second edition, North-Holland Publishing Comp., 1979. MR0344507 (49:9246)

[10] H. Matsumura, Commutative Algebra, Second edition, Math. Lecture Note Series 56, Benjamin/Cummings, London Amsterdam Tokyo, 1980. MF0575344 (82i:13003)

[11] J. Milnor, On axiomatic homology theory, Pacific J. Math. 12 (1962), 337-341. MR0159327 $(28: 2544)$

[12] M. Shiota, Geometry of Subanalytic and Semialgebraic Sets, Birkhäuser, Boston Basel Berlin, 1997. MR1463945 (99b:14061)

[13] Y.-T. Siu, Noetherianness of rings of holomorphic functions on Stein compact series, Proc. Amer. Math. Soc. 21 (1969), 483-489. MR0247135 (40:404) 
[14] R. Swan, Vector bundles and projective modules, Trans. Amer. Math. Soc. 105 (1962), 264277. MR0143225 (26:785)

[15] R. Swan, Topological examples of projective modules, Trans. Amer. Math. Soc. 230 (1977), 201-234. MF 0448350 (56:6657)

Max-Planck-Institut für Mathematik, Vivatsgasse 7, 53111 Bonn, Germany - and Department of Mathematics and Statistics, University of New Mexico, Albuquerque, New Mexico 87131-1141

E-mail address: kucharz@math.unm.edu 